\title{
Ion-Beam Sculpting Time Scales
}

\section{Citation}

Stein, Derek, Jiali Li, and Jene A. Golovchenko. 2002. “Ion-Beam Sculpting Time Scales."

Physical Review Letters 89 (27) (December). doi:10.1103/physrevlett.89.276106.

\section{Published Version}

doi:10.1103/PhysRevLett.89.276106

\section{Permanent link}

http://nrs.harvard.edu/urn-3:HUL.InstRepos:21976373

\section{Terms of Use}

This article was downloaded from Harvard University's DASH repository, and is made available under the terms and conditions applicable to Other Posted Material, as set forth at http:// nrs.harvard.edu/urn-3:HUL.InstRepos:dash.current.terms-of-use\#LAA

\section{Share Your Story}

The Harvard community has made this article openly available.

Please share how this access benefits you. Submit a story.

\section{Accessibility}




\title{
Ion-Beam Sculpting Time Scales
}

\author{
Derek Stein, ${ }^{1}$ Jiali $\mathrm{Li}^{2}{ }^{2}$ and Jene A. Golovchenko ${ }^{1,2}$ \\ ${ }^{1}$ Division of Engineering and Applied Sciences, Harvard University, Cambridge, Massachusetts 02138 \\ ${ }^{2}$ Department of Physics, Harvard University, Cambridge, Massachusetts 02138
}

(Received 25 July 2002; published 20 December 2002)

\begin{abstract}
A study of ion sculpting dynamics in $\mathrm{SiO}_{2}$ and $\mathrm{SiN}$ using periodically pulsed ion beams reveals material transport that depends strongly on the time structure of the pulsed beams. It is found that significant nanoscale matter transport can occur over second long time scales after the ion beam has been extinguished. A simple phenomenological model described the dynamics of ion beam sculpting in terms of two material time scales. The model accounts for the surprising observation of enhanced matter transport affected by pulsed ion beams over continuous ion beam exposure.
\end{abstract}

DOI: 10.1103/PhysRevLett.89.276106

Kiloelectronvolt ion beams induce atomic scale rearrangements when they are incident on a material surface by processes that include sputter erosion, atomic displacement, ion implantation, surface diffusion, and viscous flow [1-11]. The controllable, nanometer-scale nature of these changes make ion beams an interesting candidate for use as a nanofabrication tool. We recently reported on how low-energy ion beams can be used to fashion nanometer sized holes, or "nanopores," in silicon nitride membranes [12]. There it was shown that an ion beam incident on a material surface stimulates the lateral transport of matter into a preexisting hole, resulting in the inward growth of a thin film from the hole boundary that can ultimately form an extremely small hole in a freestanding membrane when the process is terminated at the appropriate time. We refer to this stimulated mass transport process as ion beam sculpting to differentiate it from sputter erosion.

Here we describe experiments using periodically pulsed ion beams that directly probe the time scales for materials transport on ion sculpted silicon dioxide and silicon nitride. The measurements are the first demonstration that ion sculpting can be successfully applied to the technologically important oxide of silicon, and they show transport time scales that are surprisingly long, indicating that these materials do not relax to their equilibrium condition until seconds after the stimulating ion beam has been extinguished.

Samples for ion beam sculpting [Fig. 1(a)] were prepared as $500 \mathrm{~nm}$ thick, freestanding $\mathrm{SiO}_{2}$ or $\mathrm{SiN}$ membranes supported on a silicon frame. The $\mathrm{SiO}_{2}$ membranes were thermally grown on $\mathrm{Si}(100)$ substrates, then capped for further processing by depositing $200 \mathrm{~nm}$ of $\mathrm{Si}_{3} \mathrm{~N}_{4}$ by low pressure chemical vapor deposition (Silicon Quest International). Photolithography and anisotropic wet chemical etching of silicon were used to create the freestanding $90 \mu \mathrm{m} \times 90 \mu \mathrm{m}$ membranes. The $\mathrm{Si}_{3} \mathrm{~N}_{4}$ capping layer was removed by etching in hot phosphoric acid, and then the oxide surface was cleaned by exposing it to an oxygen plasma. Silicon nitride membranes were
PACS numbers: 68.49.Sf, 61.72.Ff, 61.72.Qq, 68.65.-k

fabricated by first depositing $500 \mathrm{~nm}$ of low stress $\mathrm{SiN}$ directly on $\mathrm{Si}$ (100) substrates by low pressure chemical vapor deposition (LPCVD) followed by similar photolithography and etching steps as used on the oxide. A single initial hole of $\sim 70 \mathrm{~nm}$ diam was then milled through the center of the $\mathrm{SiO}_{2}$ or $\mathrm{SiN}$ membranes using a focused ion beam (FIB) machine.

The apparatus used to fabricate nanopores and study ion-stimulated material transport is shown in Fig. 1(b). A $3 \mathrm{keV}$ argon ion beam impinges on the sample surface in the normal direction. Sample surface charge neutrality is maintained using a low-energy electron beam. The ions transmitted through the open hole are detected by a Channeltron single ion detector after electrostatic focusing and energy selection. Because the transmitted ion current is directly proportional to the area of the hole, the ion beam flux can be determined by dividing the initial ion beam count rate by the initial hole size, as measured by transmission electron microscopy (TEM). The periodic time structure of pulsed ion beams is controlled by electrostatically deflecting the beam on and off the sample for the desired "on" and "off" times $\left(T_{\text {on }}\right.$ and $T_{\text {off }}$, respectively) that are indicated in the experimental results and equations presented below. Pulses of $200 \mathrm{~V}$

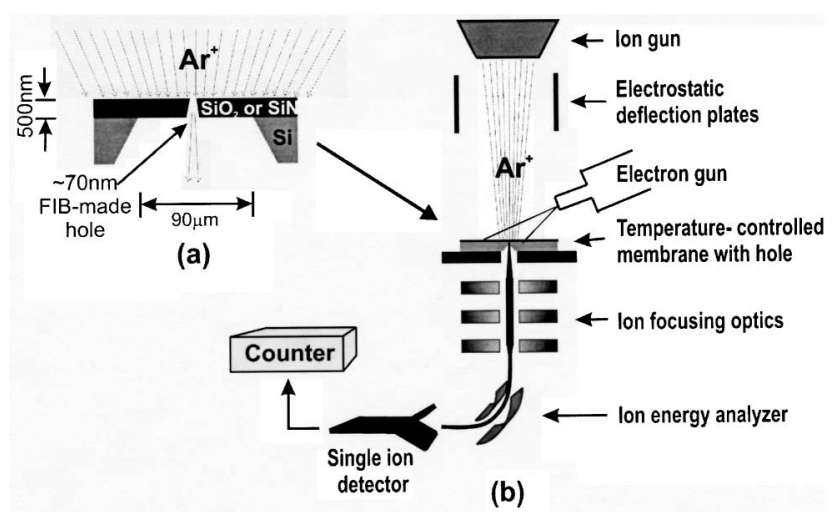

FIG. 1. (a) Schematic cross section of a $\mathrm{SiO}_{2}$ or low-stress $\mathrm{SiN}$ sample. (b) Schematic of the ion beam sculpting apparatus. 
applied to deflection plates at the exit port of the ion gun steer the beam $\sim 5 \mathrm{~mm}$ away from the hole within one $\mu \mathrm{s}$.

The effect of the ion beam time structure on ion beam sculpting dynamics is illustrated by the closing rate of a $55 \mathrm{~nm}$ hole in $\mathrm{SiO}_{2}$, at $28{ }^{\circ} \mathrm{C}$ exposed to a pulsed beam with a range of $T_{\text {off }}$ shown in Fig. 2(a). In this experiment, the flux during $T_{\text {on }}$ was kept constant at $15.7 \mathrm{Ar}^{+} \mathrm{nm}^{-2} \mathrm{~s}^{-1}$ and $T_{\text {on }}$ was $0.1 \mathrm{~s}$ per cycle throughout the experiment. The initial duty cycle of the beam was $T_{\text {on }}=0.1 \mathrm{~s}, T_{\text {off }}=0.9 \mathrm{~s}$. Without interrupting the experiment, $T_{\text {off }}$ was changed to $0.1,1.9,0.1,0$ (continuous exposure), 0.9, 0.1, and then $0.4 \mathrm{~s}$ for 100 cycles each. The hole area clearly decreases with ion beam exposure at a rate that depends on $T_{\text {off }}$. Whereas the continuous beam tends to open the hole, remarkably a pulsed beam with $T_{\text {off }} \geq 0.1 \mathrm{~s}$ acts to close it. We refer to the hole area decrease per unit fluence of the incident ion beam as the hole closing efficiency. This is the negative slope in Fig. 2(a). It is clearly an increasing function of $T_{\text {off }}$ up to 1-2 $\mathrm{s}$. The ion beam exposure can be terminated when the ion transmission rate through the hole reaches an appropriate threshold that results in a hole of the desired size with nanometer precision. The TEM image of a

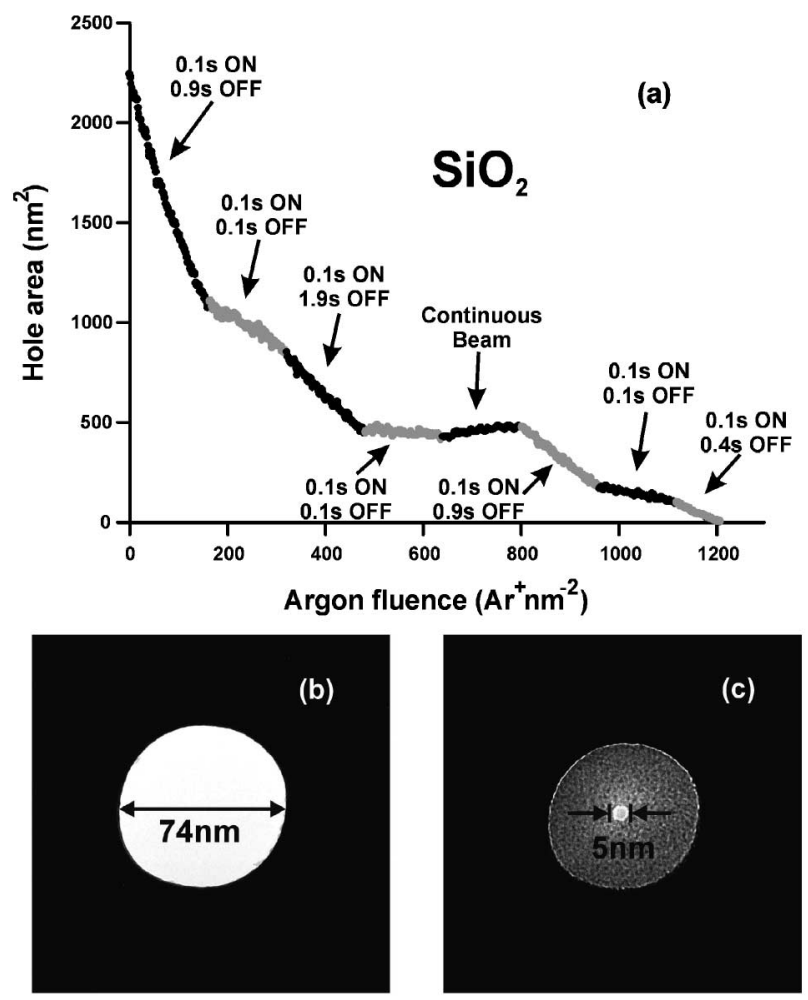

FIG. 2. (a) $T_{\text {off }}$ dependence of ion beam sculpting in $\mathrm{SiO}_{2}$. The area of a hole in a $\mathrm{SiO}_{2}$ membrane is plotted as a function of fluence from a periodically pulsed, $15.7 \mathrm{Ar}^{+} \mathrm{nm}^{-2} \mathrm{~s}^{-1}$ ion beam. $T_{\text {on }}$ was held constant at $0.1 \mathrm{~s}$ while $T_{\text {off }}$ was varied as indicated. A before (b) and after (c) TEM image of a typical, $74 \mathrm{~nm}$ wide, FIB made hole in $\mathrm{SiO}_{2}$ that was made to controllably shrink to $5 \mathrm{~nm}$ by low-energy ion irradiation. typical FIB made hole in $\mathrm{SiO}_{2}$ membrane is presented in Fig. 2(b). The ion-stimulated thin film growth over this hole was terminated such that the $5 \mathrm{~nm}$ nanopore shown in Fig. 2(c) was created.

Results for a $\mathrm{SiN}$ hole reveal a very similar duty-cycle dependence to $\mathrm{SiO}_{2}$. Figure 3 shows the closing efficiency of a $100 \mathrm{~nm}$ hole in $\mathrm{SiN}$ at $28^{\circ} \mathrm{C}$, measured for a variety of $T_{\text {off }}$ with a flux of $11.2 \mathrm{Ar}^{+} \mathrm{nm}^{-2} \mathrm{~s}^{-1}$ during $T_{\text {on }}$. The hole closing efficiency clearly increased $T_{\text {off }}$ was increased from 0.5 through 1.5 and $3.5 \mathrm{~s}$ and was unchanged at $5.5 \mathrm{~s}$ while $T_{\text {on }}$ was held constant at $0.5 \mathrm{~s}$. Note also that continuous ion beam exposure opened the hole at $28^{\circ} \mathrm{C}$. The lateral transport of matter can be "frozen out" at low temperatures to provide a measure of the sputter erosion pore opening rate. The last data segment of Fig. 3 shows that the $T_{\text {on }}=0.1 \mathrm{~s}, T_{\text {off }}=0.9 \mathrm{~s}$ pulsed beam that closed the hole at $28{ }^{\circ} \mathrm{C}$ increased its radius at a rate of $1.06 \times$ $10^{-2} \mathrm{~nm} /\left(\mathrm{Ar}^{+} \mathrm{nm}^{-2}\right)$ at $-100^{\circ} \mathrm{C}$.

We have found that the closing efficiency measured at a fixed flux and temperature can be influenced by the initial condition of the hole, including its size and surface topography for both $\mathrm{SiO}_{2}$ and $\mathrm{SiN}$. A large initial hole, $\sim 150 \mathrm{~nm}$ for example, can be opened under the same conditions that would close a smaller, $\sim 50 \mathrm{~nm}$, hole. The closing efficiency can be enhanced to promote hole closing by lowering the ion flux or raising the temperature. The duty-cycle dependence of the closing efficiency remains an increasing function of $T_{\text {off }}$ regardless of flux, temperature, or initial condition of the hole.

On the microscopic scale, ion beam sculpting may involve the motion of independent atoms along the surface of the material or the collective motion of many atoms in a surface layer to account for the lateral transport of matter under the influence of low-energy ion

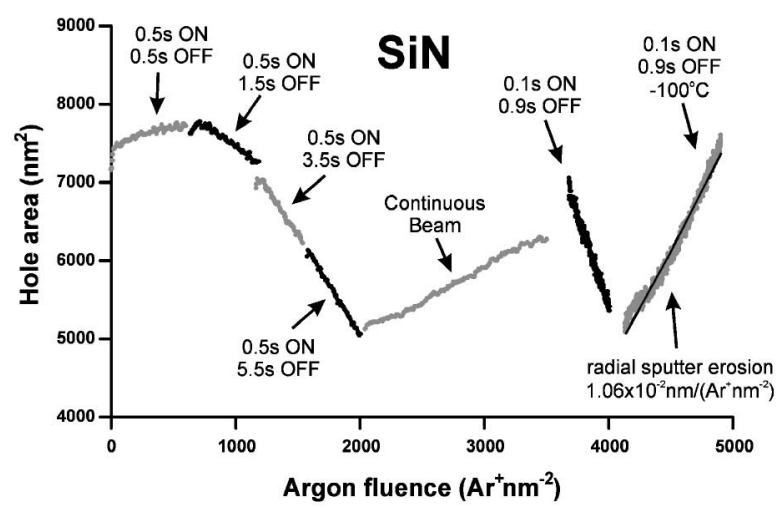

FIG. 3. Duty cycle dependence of ion sculpting in SiN. The area of a hole in a SiN membrane is plotted as a function of fluence from a periodically pulsed, $11.2 \mathrm{Ar}^{+} \mathrm{nm}^{-2} \mathrm{~s}^{-1}$ ion beam. The duty cycle corresponding to each segment of the curve is indicated by $T_{\text {on }}$ and $T_{\text {off }}$. The hole opening effect of sputter erosion is measured in the final (rightmost) segment by reducing the sample temperature to $-100^{\circ} \mathrm{C}$, where the lateral transport of matter is frozen out. 
beams. Elements of these two views, embodied in surface diffusion [7,8] and viscous flow models [10,11,13-15], may be needed for a full understanding of the ion beam sculpting phenomenon. To describe the time-dependent behavior of ion beam sculpting here, at first ignoring the effect of sputter erosion, we parametrize material response with a rise time, $\tau_{\text {rise }}$, and decay time, $\tau_{\text {decay }}$. Within this time scale description the closing rate of the hole approaches a steady-state (continuous beam) value $(d A / d t)_{\mathrm{ss}}$ as $\left[1-e^{-t / \tau(\text { rise })}\right](d A / d t)_{\mathrm{ss}}$ when the ion beam is turned on, and decays as $e^{-t / \tau(\text { decay })}$ when the beam is turned off. An exponential rise and decay of the hole closing rate is the simplest phenomenological model that incorporates material response time scales. Such time scales are common to microscopic models that are the most likely candidates to describe the ion sculpting phenomenon, including surface diffusion or viscous flow models. We require that the closing rate be a continuous function of time that we assume reaches a quasisteady state condition where the closing rate at the beginning of each pulse (time $t=0$ in the equations below) is the same. Under these conditions the closing rate of the hole area, $A$, is given by

$$
\begin{aligned}
& \frac{d A}{d t}=\left(\frac{d A}{d t}\right)_{\mathrm{ss}}\left[1-e^{-t / \tau_{\text {rise }}}\left(\frac{1-e^{-T_{\text {off }} / \tau_{\text {decay }}}}{1-e^{-\left(T_{\text {off }} / \tau_{\text {decay }}\right)-\left(T_{\text {on }} / \tau_{\text {rise }}\right)}}\right)\right], \quad 0 \leq t<T_{\text {on }}, \\
& \frac{d A}{d t}=\left(\frac{d A}{d t}\right)_{\mathrm{ss}}\left[1-e^{-T_{\text {on }} / \tau_{\text {rise }}}\left(\frac{1-e^{-T_{\text {off }} / \tau_{\text {decay }}}}{1-e^{-\left(T_{\text {off }} / \tau_{\text {decay }}\right)-\left(T_{\text {on }} / \tau_{\text {rise }}\right)}}\right)\right] e^{-\left(t-T_{\text {on }}\right) / \tau_{\text {decay }}}, \quad T_{\text {on }} \leq t \leq T_{\text {off }} .
\end{aligned}
$$

A hole closes over the entire beam pulse cycle, therefore the total area change induced by the ion beam must be integrated over $T_{\text {on }}$ [Eq. (1a)] and $T_{\text {off }}$ [Eq. (1b)]. The theoretical closing efficiency in the absence of sputter erosion, $\xi$, at flux $\phi$ becomes

$$
\xi=\frac{1}{\phi T_{\text {on }}} \int_{\text {cycle }} \frac{d A}{d t} d t=\frac{1}{\phi}\left(\frac{d A}{d t}\right)_{\text {ss }}\left[1+\frac{\left(\tau_{\text {decay }}-\tau_{\text {rise }}\right)}{T_{\text {on }}} \frac{\left(1-e^{\left.-T_{\text {on }} / \tau_{\text {rise }}\right)}\right)\left(1-e^{-T_{\text {off }} / \tau_{\text {decay }}}\right)}{1-e^{-\left(T_{\text {on }} / \tau_{\text {rise }}\right)-\left(T_{\text {off }} / \tau_{\text {decay }}\right)}}\right] .
$$

Equation (2) accounts for duty-cycle dependence in ion beam sculpting through the relative lengths of the material response time scales. The experimental results presented in Figs. 2 and 3 suggest that the intrinsic (beam off) $\tau_{\text {decay }}$ of both $\mathrm{SiO}_{2}$ and $\mathrm{SiN}$ is 1-2 sec, and fitting the results to the exponential time scale model predicts that $\tau_{\text {rise }}$ must be shorter than $\tau_{\text {decay }}$.

A microscopic model for ion beam sculpting based on the surface diffusion of ion-stimulated mobile species [12] predicts that $\tau_{\text {rise }}$ decreases with increasing flux. The mechanism relies on incoming ions ejecting mobile surface species, thereby reducing the intrinsic time such species can contribute to matter transport. No detailed model has yet been developed to describe ion beam sculpting in terms of ion-induced viscous flow. It is unlikely that any microscopic model based on the sputter redeposition of matter could account for the time scales involved in ion beam sculpting. Ion sputter erosion is known to arise from momentum transfer in atomic collision cascades that last on the order of a picosecond $[16,17]$. The time scale for a material to respond in ion beam sculpting is unusually long-on the order of seconds.

The extent to which the ion beam reduces $\tau_{\text {rise }}$ relative to $\tau_{\text {decay }}$ can be tested within the time scale model by measuring the ratio of $\xi$ for a continuous to a pulsed beam. The expression for $\xi$ from Eq. (2) is approximated by $(d A / d t)_{\mathrm{ss}} \phi^{-1}\left[1+\left(\tau_{\text {decay }}-\tau_{\text {rise }}\right)\left(\tau_{\text {decay }}+\tau_{\text {rise }}\right)^{-1}\right]$ for a pulsed beam where $T_{\text {on }}$ and $T_{\text {off }}$ are the same and much shorter than $\tau_{\text {decay }}$ or $\tau_{\text {rise }}$. The hole closing efficiency for a continuous beam is $(d A / d t)_{\mathrm{ss}} \phi^{-1}$. As $\tau_{\text {rise }}$ decreases, the closing efficiency of the pulsed beam should tend to twice that value, regardless of the material. This prediction is surprising because it implies that the same amount of hole closing can be induced by half the ion fluence in the same amount of time by using a periodically pulsed beam.

Figure 4 shows the closing efficiency of a quickly pulsed beam relative to a continuous beam for $\mathrm{SiO}_{2}$ and $\mathrm{SiN}$. The experiment was performed by switching between continuous ion beam exposure and a periodically pulsed beam with $T_{\text {on }}=T_{\text {off }}=10 \mathrm{~ms}$. The incident ion flux was $8.3 \mathrm{Ar}^{+} \mathrm{nm}^{-2} \mathrm{~s}^{-1}$ for the nitride sample and $2.2 \mathrm{Ar}^{+} \mathrm{nm}^{-2} \mathrm{~s}^{-1}$ for the oxide sample. The closing efficiency was clearly enhanced by the periodically pulsed beams in both materials. For $\mathrm{SiO}_{2}$, the measured value in units of $\mathrm{nm}^{2} /\left(\mathrm{Ar}^{+} \mathrm{nm}^{-2}\right)$ increased from 0.3 (continuous beam) to 1.4 (pulsed beam). The measurement was repeated on the same $\mathrm{SiO}_{2}$ sample to find an increase in closing efficiency from 0.5 (continuous beam) to 1.3 (pulsed beam). The measured closing efficiency for SiN increased from 1.6 (continuous beam) to 5.8 (pulsed beam), then returned to 3.5 when the continuous beam measurement was repeated.

The time scale model predicts $\xi$ should be enhanced for a pulsed beam versus a continuous beam by a factor of 2 if $\tau_{\text {rise }} \ll \tau_{\text {decay }}$, and again neglecting sputter erosion effects. The observed closing efficiency enhancement factors of 3.4 for the $\mathrm{SiO}_{2}$ sample, and 2.3 for the $\mathrm{SiN}$ sample were obtained by averaging the slopes for continuous or pulsed segments of the data from the beginning and the 


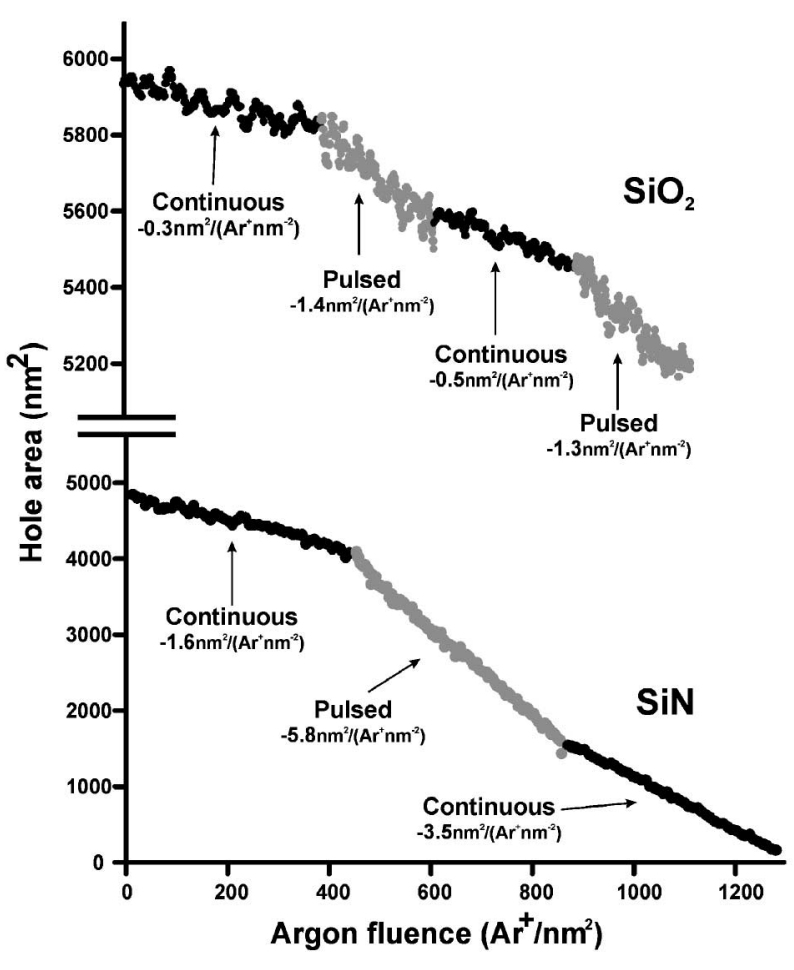

FIG. 4. Comparison of ion sculpting efficiencies of pulsed and continuous ion beams in $\mathrm{SiN}$ and $\mathrm{SiO}_{2}$. The area change of a hole induced per unit fluence is measured to be greater for a $T_{\text {on }}=T_{\text {off }}=10 \mathrm{~ms}$ periodically pulsed ion beam than for continuous ion beam exposure in both $\mathrm{SiO}_{2}$ and low stress $\mathrm{SiN}$, as indicated on the curves.

end of each experiment. These likely differ due to a radius-dependent sputter erosion contribution and surface material changes induced by significant argon irradiation. To compare the measured hole closing efficiencies with $\xi$, we must now subtract the competing effect of pore opening caused by sputter erosion from the experimental data by using the low temperature data in Fig. 3. Sputter erosion is assumed to contribute a constant radial hole opening rate of $1.06 \times 10^{-2} \mathrm{~nm} /\left(\mathrm{Ar}^{+} \mathrm{nm}^{-2}\right)$ since the sputter yields of $\mathrm{SiO}_{2}$ and $\mathrm{SiN}$ are very similar [18], weakly flux dependent [19], and presumed to be independent of duty cycle. The experimental $\xi$-enhancement factors are 1.3 for the $\mathrm{SiO}_{2}$ experiment and 1.8 for the $\mathrm{SiN}$ experiment. These values are still less than the predicted value of 2 because $\tau_{\text {rise }}$, although significantly diminished relative to $\tau_{\text {decay }}$, is not negligibly small. From Eq. (2), we calculate that $\tau_{\text {rise }}$ is reduced to 0.6 times $\tau_{\text {decay }}$ for $\mathrm{SiO}_{2}$ by a $2.2 \mathrm{Ar}^{+} \mathrm{nm}^{-2} \mathrm{~s}^{-1}$ ion beam. Similarly, an $8.3 \mathrm{Ar}^{+} \mathrm{nm}^{-2} \mathrm{~s}^{-1}$ ion beam reduces $\tau_{\text {rise }}$ to one eighth $\tau_{\text {decay }}$ for $\mathrm{SiN}$.

In conclusion, we have shown that the dynamics of hole closing in $\mathrm{SiO}_{2}$ and $\mathrm{SiN}$ are strongly dependent on the rest time between ion beam pulses, implying that a significant amount of hole closing occurs when no beam is incident on the material surface. The effect can be described by an exponential rise and decay of the hole closing rate. This work is relevant to nanofabrication because ion beam time structure affects the degree of matter transport induced by a given dose. It can even determine the direction of transport by influencing the competition between ion-induced lateral matter transport and sputter erosion processes. We already exploit these effects in the fabrication of solid-state nanopores used in single-molecule biological studies.

The authors wish to acknowledge stimulating discussion and technical assistance from Michael J. Aziz and Bola George. This work was supported by the National Science Foundation through Grant No. DMR-0073590, DARPA through Grant No. F49620-01-1-0478, the Department of Energy through Grant No. DE-FG0201ER45922, and Agilent Technologies.

[1] H. Gnaser, Ion Irradiation of Solid Surfaces (Springer, Berlin, 1999).

[2] R. E. Johnson and J. Shou, K. Dan. Vidensk. Selsk. Mat. Fys. Medd. 43, 403 (1993).

[3] P. Sigmund, K. Dan. Vidensk. Selsk. Mat. Fys. Medd. 43, 7-26 (1993).

[4] T. Nenandovic, B. Peraillon, Z. Bogdanov, Z. Djordjevic, and M. Millic, Nucl. Instrum. Methods Phys. Res., Sect. B 48, 538 (1990).

[5] P. G. Snyder, A. Massengale, K. Memarsadeh, J. A. Woolam, D. C. Ingram, and P. P. Pronko, Mater. Res. Soc. Symp. Proc. 74, 535 (1987).

[6] E. A. Eklund, R. Bruinsma, J. Rudnick, and R.S. Williams, Phys. Rev. Lett. 67, 1759 (1991).

[7] J. Erlebacher, M. J. Aziz, E. Chason, M. B. Sinclair, and J. A. Floro, Phys. Rev. Lett. 82, 2330 (1999).

[8] J. Erlebacher, M. J. Aziz, and E. Chason, J. Vac. Sci. Technol. A 18, 115 (2000).

[9] K Oyoshi, T. Tayami, and S. Tanaka, Jpn. J. Appl. Phys. 30, 1854 (1991).

[10] T. M. Mayer, E. Chason, and A. J. Howard, J. Appl. Phys. 76, 1633 (1994).

[11] C. C. Umbach, R. L. Headrick, and K.-C. Chang, Phys. Rev. Lett. 87, 246104 (2001).

[12] J. Li, D. Stein, C. McMullan, D. Branton, M. J. Aziz, and J. A. Golovchenko, Nature (London) 412, 166 (2001).

[13] G. Carter, Surf. Interface Anal. 25, 952 (1997).

[14] M. L. Brongersma, E. Snoeks, T.V. Dillen, and A. Polman, J. Appl. Phys. 88, 59 (2000).

[15] H. Trinkaus and A. I. Ryazanov, Phys. Rev. Lett. 74, 5072 (1995).

[16] T. Diaz de la Rubia, R. S. Averback, R. Benedek, and W. E. King, Phys. Rev. Lett. 59, 1930 (1987).

[17] R. S. Averback and T. Diaz de la Rubia, in Solid State Physics, edited by H. Ehrenreich and F. Spaepen (Academic Press, San Diego, 1998), Vol. 51, p. 281.

[18] J. F. Ziegler and J. P. Biersack, Report No. SRIM-2000, available at http://www.srim.org

[19] G. Carter, J. Phys. D 34, R1 (2001). 